Research Article

\title{
Simulation of Train-Turnout Coupled Dynamics Using a Multibody Simulation Software
}

\author{
Albert Lau $(\mathbb{D})$ and Inge Hoff $(1)$ \\ Department of Civil and Environmental Engineering, Norwegian University of Science and Technology, Trondheim, Norway \\ Correspondence should be addressed to Albert Lau; albert.lau@ntnu.no
}

Received 7 December 2017; Revised 18 May 2018; Accepted 24 June 2018; Published 22 July 2018

Academic Editor: Gaetano Sequenzia

Copyright ( $\odot 2018$ Albert Lau and Inge Hoff. This is an open access article distributed under the Creative Commons Attribution License, which permits unrestricted use, distribution, and reproduction in any medium, provided the original work is properly cited.

\begin{abstract}
With the advancements of computing power, multibody simulation (MBS) tool is used to study not only train dynamics but also more realistic phenomena such as train-track coupled dynamics. However, train-turnout coupled dynamics within MBS is still hard to be found. In this paper, a train-turnout coupled model methodology using a MBS tool GENSYS is presented. Dynamic track properties of a railway track are identified through numerical receptance test on a simple straight track model. After that, the identified dynamic track properties are adopted in a switch and crossing (turnout) to simulate train-turnout coupled dynamic interaction including parameters such as rail bending stiffness and sleeper mass variation along the turnout. The train-turnout coupled dynamic interaction is compared to the dynamic interaction simulated from a widely accepted moving mass trainturnout model. It is observed that the vertical and lateral normal forces for the new train-turnout coupled model and the conventional moving mass train-turnout model are in good agreement. In addition, the new train-turnout coupled model can provide additional track dynamics results. It is concluded that the train-turnout coupled model can provide a more realistic trainturnout dynamic interaction compared to the moving mass train-turnout model.
\end{abstract}

\section{Introduction}

A good understanding of the train and track dynamic interaction is important to optimise design and maintenance of the track components. Switches and crossings are of special interest because of the increased loading that causes extra wear and high maintenance costs. Train and track dynamic simulations are used to study how train or track reacts when they are subjected to different conditions such as different axle loads, travelling speed, and track geometry especially at the design and development stages. Through train and track dynamic analysis, it is possible to evaluate how modifications in train and/or track affect several factors such as riding comfort, vehicle or track accelerations, derailment risk, rolling contact fatigue, rail stresses, and rail deflection. On top of that, numerical simulations reduce reliance on experimental campaigns that in most cases can be very expensive and time consuming.

Two types of numerical methods are most commonly used in the quest of simulating train and/or track dynamics, that is, multibody simulation (MBS) method and finite element method (FEM). Since the train-track complicated when it comes to dynamics, geometry, and material behaviour, it is necessary to make simplifications to be able to build the model and run the simulations in a reasonable time. The MBS method is known to be efficient to study the train dynamics, but the tradeoff is the track model that has to be simplified. In the FEM, a very detailed train-track model can be modelled including bodies' structural flexibility; however, the computational effort is much higher compared to MBS. Therefore, FEM in railway field is often used only for very detailed component modelling [1], detailed wheel-rail contact modelling [2,3], and so on. Sometimes, FEM is also used for train-track simulation as a whole, but simplifications are often made to reduce the computational effort $[4,5]$.

MBS is preferable to study train-track dynamic interaction as a whole, especially for the complex railway track component such as switch and crossing. MBS tool dedicated for train-track analysis has a wheel-rail contact module that is able to model complex wheel-rail kinematic behaviour and the corresponding creep forces. Some authors, for example, 
Kassa and Nielsen [6], Lagos et al. [7], Wan et al. [8], Pålsson [9], and Lau and Kassa [10], used MBS to simulate trainturnout dynamic interaction to evaluate the maximum contact forces along a railway turnout, to study the influence of the turnout geometry, to optimise crossing nose profile, to optimise switch geometry, and to study the effect of yaw in a switch and crossing, respectively. As reported by Esveld [11], the track substructure has a direct influence on the dynamic wheel load, dynamic track stiffness, and track roughness. However, modelling railway track in MBS has always been a challenge [12]. Moreover, the train-turnout model used by the abovementioned authors is moving mass train-turnout model of which the track is represented by a lumped mass or mass layers coupled using spring-damper elements, and the track follows the train's wheels throughout the simulation. Such track model is able to capture dynamics up to $200 \mathrm{~Hz}$. Some frequencies such as sleeper passing frequency and frequencies induced by the rail bending stiffness are not able to be modelled. Moreover, constant sleeper mass is used even though reality sleeper mass is not the same along switches and crossings.

In the present paper, a train-turnout coupled model using a MBS software GENSYS is presented. Dynamic track properties are first determined using a simple straight track model through the receptance test by referring to existing receptance functions recorded in the literature. After that, the determined track properties are applied to a turnout model. The train-turnout coupled model is simulated, and the corresponding dynamic interaction is compared to the dynamic interaction from a moving mass train-turnout model. Some track dynamics of the train-turnout coupled model are also reported. Benefits and drawbacks of the model are discussed in the paper.

\section{Train Model}

Train model presented in this paper is a Norwegian passenger train represented by seven bodies, that is, a carbody, two bogies, and four wheelsets. Each of the bodies has six degrees of freedom (DOFs), that is, three translations and three rotations apart from the wheelsets which have only five DOFs with longitudinal translation being constrained. The bodies are coupled to each other with spring-damper elements. Unlike conventional moving mass model, the train model in this paper is modelled in a moving coordinate system relative to the track fixed coordinate system. Figure 1 illustrates a schematic drawing of the train and the track model.

\section{Turnout Model}

3.1. Track Modelling. There have been some attempts to model railway track using MBS, for example, in [13-15]. One of the most popular ways of modelling railway track is modelling rail of the track as a beam element using the Euler-Bernoulli beam theory. State of the general dynamic beam equation, rail in this case, can be expressed by the Euler-Lagrange equation:

$$
L=\frac{1}{2} \rho A\left(\frac{\partial w}{\partial t}\right)^{2}-\frac{1}{2} E I\left(\frac{\partial^{2} w}{\partial x^{2}}\right)^{2}+q(x, t) w(x, t),
$$

where $\rho$ is the mass density, $A$ is the cross-sectional area, $E$ is the elastic modulus, and $I$ is the second moment of the area of beam's cross section. Since Euler-Bernoulli beam can only be supplied with the load and provide deflection in vertical direction, $q(x, t)$ is the external load and $w(x, t)$ is the deflection of the beam in the vertical direction at time $t$ along the longitudinal position $x$. The external load $q(x, t)$ is the excitation and the corresponding reaction generated by the train (wheel) on the top of the beam and is expressed as

$$
\begin{aligned}
q(x, t)= & -\sum_{i=1}^{N_{\mathrm{s}}} R_{i} \delta\left(x-x_{i}\right)+\sum_{j=1}^{N_{\mathrm{w}}} F_{j} \delta\left(x-x_{j}\right) \\
& \cdot\left[H\left(t-t_{j}\right)-H\left(t-t_{j}-\Delta t_{j}\right)\right],
\end{aligned}
$$

where $N_{\mathrm{s}}$ is the number of supports, which in this case is modelled as a body rigidly connected under the beam with vertical translation and rotation in pitch direction unconstrained, $R_{i}$ is the $i$ th support reaction force, $\delta(\cdot)$ is the Dirac delta function, $x$ is the current position, $x_{i}$ is the support position, $N_{\mathrm{w}}$ is the number of wheels travelling over the beam, $F_{j}$ is the wheel-rail contact force under the $j$ th wheel, $H(\sim)$ is the unit step function, $t$ is the current time step, $t_{j}$ is the inbound time of the $j$ th wheel at the beam, and $\Delta t_{j}$ is the time travelled of the $j$ th wheel through the beam. Since

$$
\frac{\partial L}{\partial w}=\frac{\partial}{\partial t}\left(\frac{\partial L}{\partial \dot{w}}\right)+\frac{\partial^{2}}{\partial x^{2}}\left(\frac{\partial L}{\partial w_{x x}}\right)
$$

Euler-Lagrange equation can be expressed as

$$
q(x, t)=\rho A \frac{\partial^{2} w}{\partial t^{2}}+C \frac{\partial w}{\partial t}+E I \frac{\partial^{4} w}{\partial x^{4}}
$$

where $C$ is the viscous damping coefficient. Through modal superposition, the vertical deflection $w(x, t)$ can be expressed by the following equation:

$$
w(x, t)=\sum_{h=1}^{\infty} A_{h}(t) \cdot S_{z}(h, x),
$$

where $A_{h}(t)$ is the $h$ th mode time coefficient of vertical deflection and $S_{z}(h, x)$ is the $h$ th mode shape function of vertical deflection. Substituting (5) into (4) yields the following equation:

$$
\ddot{W}_{h}+2 \xi_{h} \omega_{h} \cdot \dot{W}_{h}+\omega_{h}^{2} W_{h}=\frac{q}{m_{h}}, \quad(h=1,2, \ldots, \infty),
$$

where $\omega_{h}=(h \pi / \mathrm{L})^{2} \cdot \sqrt{E I / \rho A}$ is the model frequency, $\xi_{h}=$ $C / 2 \rho A \omega_{h}$ is the damping ratio, and $m_{h}=\rho A$ is the model mass of the $h$ th mode for a single span beam.

Note that when defining the beam in GENSYS, EulerBernoulli beam function is a massless beam and the beam mass is only considered by the rail masses connected rigidly under the beam. This is because these masses are needed to act as bodies that allow more masses such as sleeper masses and ballast masses to be connected underneath them to 


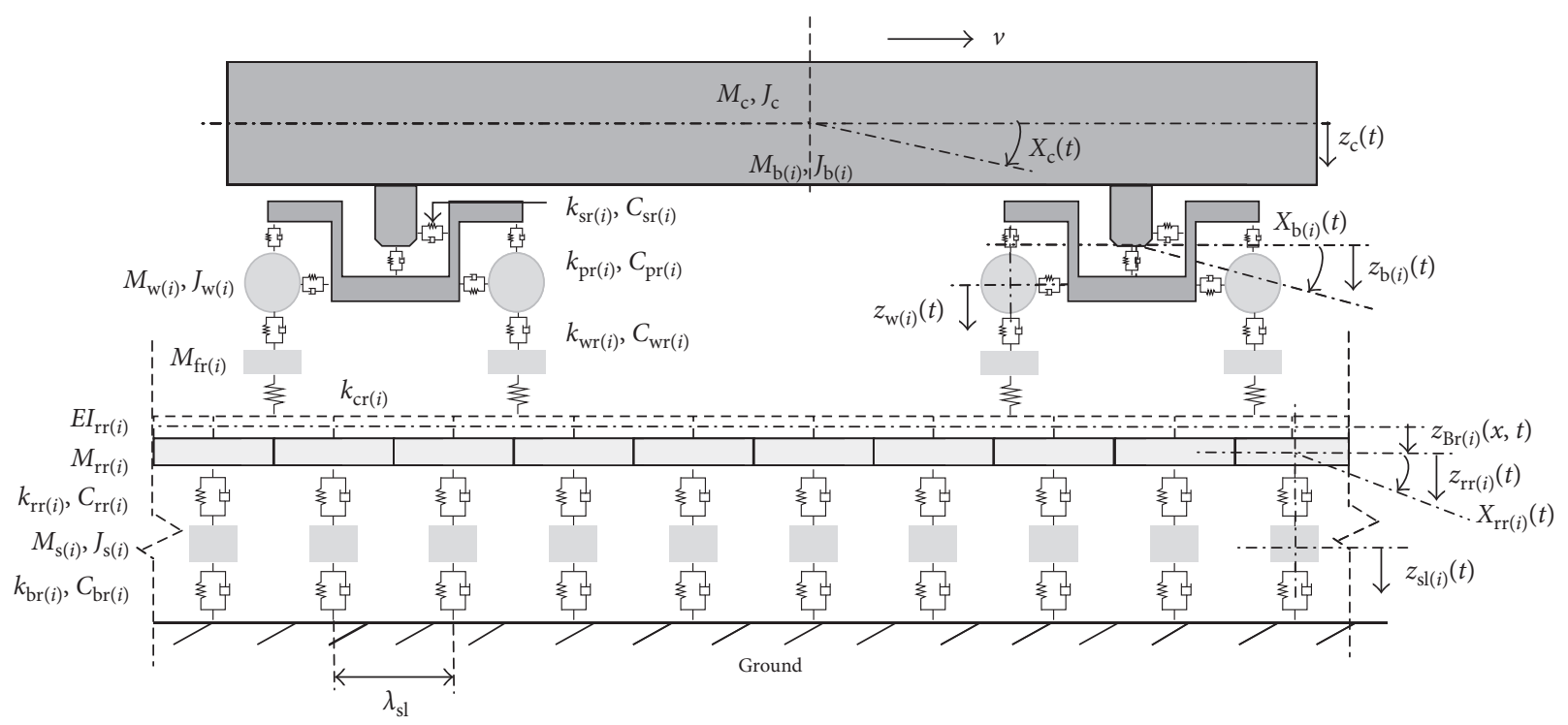

(a)

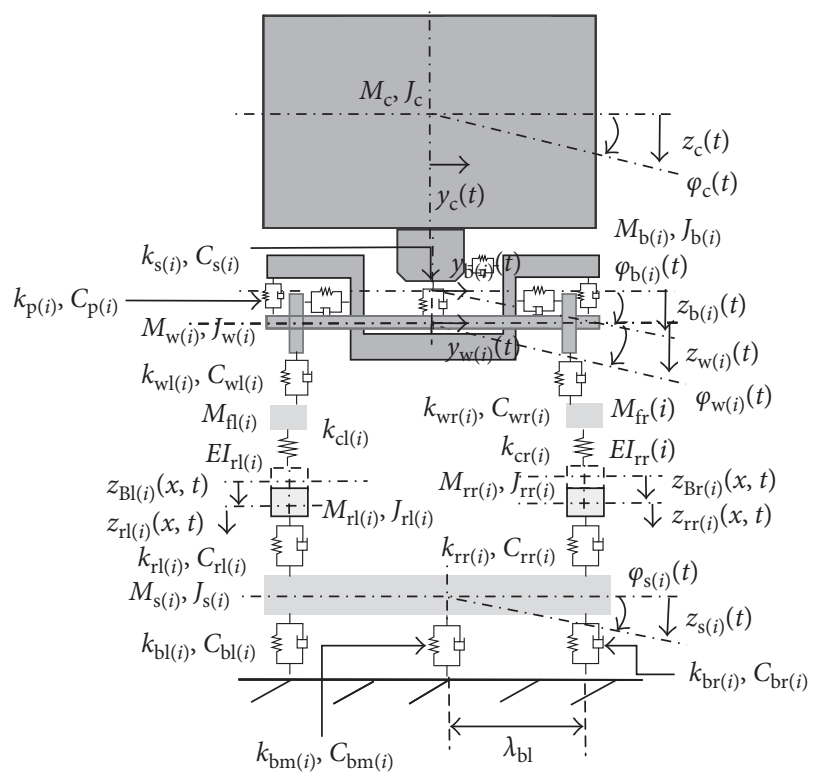

(b)

FIGURE 1: Schematic drawing of the train-track model (not to scale): (a) side view and (b) rear view. $v$ : wheelset velocity, $E I_{\mathrm{r}}$ : rail bending stiffness, second subscript $l$ : left position, $k_{\mathrm{r}}$ : railpad stiffness, second subscript $r$ : right position, $c_{\mathrm{r}}$ : railpad damping, second subscript $m$ : middle position, $k_{\mathrm{b}}$ : ballast stiffness, subscript $i$ : item number, $c_{\mathrm{b}}$ : ballast damping, $M_{\mathrm{c}}$ : carbody mass, $z_{\mathrm{c}}$ : carbody vertical displacement, $J_{\mathrm{c}}$ : carbody mass inertia, $z_{\mathrm{b}}$ : bogie vertical displacement, $M_{\mathrm{b}}$ : bogie mass, $z_{\mathrm{w}}$ : wheelset vertical displacement, $J_{\mathrm{b}}$ : bogie mass inertia, $z_{\mathrm{s}}$ : sleeper mass vertical displacement, $M_{\mathrm{w}}$ : wheelset mass, $y_{\mathrm{c}}$ : carbody lateral displacement, $J_{\mathrm{w}}$ : wheelset mass inertia, $y_{\mathrm{b}}$ : bogie lateral displacement, $M_{\mathrm{f}}$ : fictitious rail mass, $y_{\mathrm{w}}$ : wheelset lateral displacement, $M_{\mathrm{r}}$ : rail mass, $z_{\mathrm{B}}$ : rail (beam) vertical displacement, $J_{\mathrm{r}}$ : rail mass inertia, $z_{\mathrm{r}}$ : rail mass vertical displacement, $M_{\mathrm{s}}$ : sleeper mass, $\varphi_{\mathrm{c}}$ : carbody roll rotation, $J_{\mathrm{s}}$ : sleeper mass inertia, $\varphi_{\mathrm{b}}$ : bogie roll rotation, $k_{\mathrm{s}}$ : secondary suspension stiffness, $\varphi_{\mathrm{w}}$ : wheelset roll rotation, $c_{s}$ : secondary suspension damping, $\varphi_{s}$ : sleeper mass roll rotation, $k_{\mathrm{p}}$ : primary suspension stiffness, $\chi_{\mathrm{c}}$ : carbody mass pitch rotation, $c_{\mathrm{p}}$ : primary suspension damping, $\chi_{\mathrm{b}}$ : bogie mass pitch rotation, $k_{\mathrm{w}}$ : wheel-fictitious rail contact stiffness, $\chi_{\mathrm{r}}$ : rail mass pitch rotation, $c_{\mathrm{w}}$ : wheel-fictitious rail contact damping, $\lambda_{\mathrm{sl}}$ : sleeper distance, $k_{\mathrm{c}}$ : fictitious rail-rail contact stiffness, and $\lambda_{\mathrm{bl}}$ : ballast support distance.

represent a more realistic track, Figure 1. Therefore, the Euler-Lagrange equation as stated in (1) is only completed when the rail masses rigidly connected under the beam are defined. The rail masses are constrained in every direction apart from vertical translation and pitch rotation. One should be careful when selecting the moment of inertia of the rail masses in pitch direction as it has direct consequences to the bending and deflection of the beam. For instance, if the moment of inertia of the rail masses is too high, the beam will be restricted from bending or the bending and the deflection of the beam will be unrealistically high if the moment of inertia is too low.

For multispan continuous beam, beam elements are represented by finite beam elements via matrix coupling. For 
more details of the finite beam elements, refer to Ottosen and Petersson [16]. If needed, for example, in switch and crossing, the bending stiffness of the beam can be varied sectionally along the track. The corresponding model frequencies, damping ratio, and model masses can be expressed by complex trigonometric functions. Such approach has previously been successfully implemented by Sun et al. [17] to simulate vertical dynamic of train-rail bridge interaction.

3.2. Track Property Validation. One of the most crucial information for the track modelling is the dynamic track properties. Although there is existing information of track properties such as the one recorded in [18], it applies only for the moving mass track model. Therefore, a numerical track receptance test is performed on a straight track model (without vehicle) to identify the dynamic properties of the track.

The straight track model for the identification consists of 100 rail masses which connects rigidly under two beams (50 on each side). Each pair of rail masses (left and right) is connected to a sleeper mass (a total of 50 sleeper masses) with spring-damper elements representing railpads. Under each sleeper, three spring-damper elements, left, middle, and right, are used to represent the ballast flexibility connecting the sleeper to the rigid ground. The arrangement of the sleeper to rigid ground coupling is such that the left and the right spring-damper elements are always $350 \mathrm{~mm}$ from the edge of the sleeper and another one is always in the middle of the sleeper. The sleeper masses are constrained in every direction apart from the vertical translation and roll rotation.

Two receptance tests, one on the rail above a sleeper and another in between two sleepers, are performed. The corresponding receptance functions are adjusted to match the receptance functions as described in the literature [19] by means of tuning the track parameters such as railpad and ballast stiffness and damping properties.

Since there is no rail mass in the middle of the supports, an approximated method has to be used to obtain the receptance function when the excitation is applied in the middle of two sleepers. The displacement of the rail in the middle of two sleepers, $\delta_{\text {mid }}$, can be approximated using the pitch angle and displacement of the adjacent rail masses:

$$
\begin{aligned}
\delta_{\text {mid }}(t)= & \delta_{i}(t) \cdot \lambda_{1}+\chi_{i}(t) \cdot x \cdot \lambda_{2}+\delta_{i+1}(t) \cdot \lambda_{3} \\
& +\chi_{i+1}(t) \cdot x \cdot \lambda_{4},
\end{aligned}
$$

where $\delta_{i}$ and $\delta_{i+1}$, and $\chi_{i}$ and $\chi_{i+1}$ are the vertical displacements and the pitch angles of the masses which rigidly connect under the beam adjacent to the point of observation, respectively, $x$ is the support distance, and $\lambda$ is the basis function which can be expressed as

$$
\begin{aligned}
& \lambda_{1}=1-3 \cdot x_{\mathrm{r}}^{2}+2 \cdot x_{\mathrm{r}}^{3}, \\
& \lambda_{2}=x_{\mathrm{r}}-2 \cdot x_{\mathrm{r}}^{2}+x_{\mathrm{r}}^{3}, \\
& \lambda_{3}=3 \cdot x_{\mathrm{r}}^{2}-2 \cdot x_{\mathrm{r}}^{3}, \\
& \lambda_{4}=-x_{\mathrm{r}}^{2}+x_{\mathrm{r}}^{3},
\end{aligned}
$$

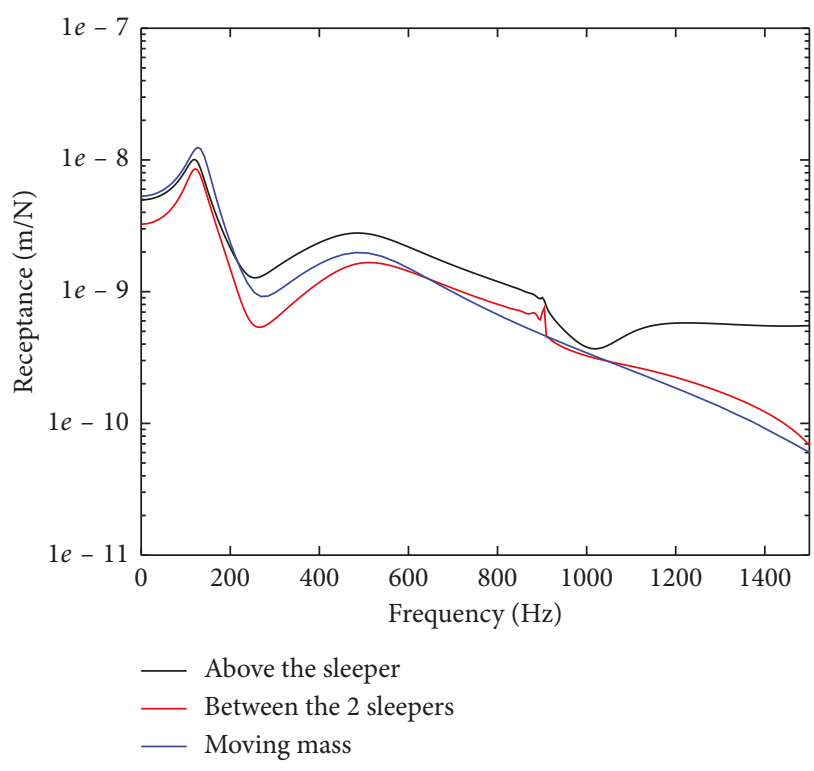

Figure 2: Receptance functions.

TABle 1: Track properties.

\begin{tabular}{lcc}
\hline Element & Unit & Value \\
\hline Euler-Bernoulli beam & Bending stiffness $\left(\mathrm{Nm}^{2}\right)$ & $6.11 \times 10^{6}$ \\
\hline \multirow{4}{*}{ Rail mass } & Mass $\left(\mathrm{kg} / \mathrm{m}^{6}\right)$ & 60 \\
& Inertia $I_{x}\left(\mathrm{kgm}^{2}\right)$ & 1.17 \\
& Inertia $I_{y}\left(\mathrm{kgm}^{2}\right)$ & 1.25 \\
& Inertia $I_{z}\left(\mathrm{kgm}^{2}\right)$ & 1.15 \\
\hline \multirow{2}{*}{ Railpad } & Stiffness $(\mathrm{N} / \mathrm{m})$ & $300 \times 10^{6}$ \\
& Damping $(\mathrm{Ns} / \mathrm{m})$ & $50 \times 10^{3}$ \\
\hline Support distance & $-(\mathrm{m})$ & 0.6 \\
\hline \multirow{3}{*}{ Sleeper mass } & Mass $(\mathrm{kg})$ & 380 \\
& Inertia $\left.I_{x}(\mathrm{kgm})^{2}\right)$ & 4.67 \\
& Inertia $\left.I_{y}(\mathrm{kgm})^{2}\right)$ & 155.09 \\
\multirow{2}{*}{ Ballast } & Inertia $\left.I_{z}(\mathrm{kgm})^{2}\right)$ & 156.12 \\
\hline
\end{tabular}

where

$$
x_{\mathrm{r}}=\frac{x_{0}}{x} \quad\left[x_{0} ¥ x\right],
$$

where $x_{\mathrm{r}}$ is the relative longitudinal position along the beam and $x_{0}$ is the distance to the position from the mass on the left of which in the case of observation in the middle of two masses, $x_{\mathrm{r}}=0.5$, and $\lambda_{1}, \lambda_{2}, \lambda_{3}$, and $\lambda_{4}$ have a value of 0.5 , $0.125,0.5$, and -0.125 , respectively.

Figure 2 shows the receptance functions of the track with the properties as listed in Table 1 . It can be seen that the three principal resonance frequencies as mentioned in [19] are captured. The first resonance, which is regarded as track resonance (rails and sleepers vibrate on the ballast) which usually occurs in the frequency range of 50 to $300 \mathrm{~Hz}$, is captured at the frequency around $129 \mathrm{~Hz}$. The second resonance frequency, which is regarded as the rails bouncing on the railpads and usually 


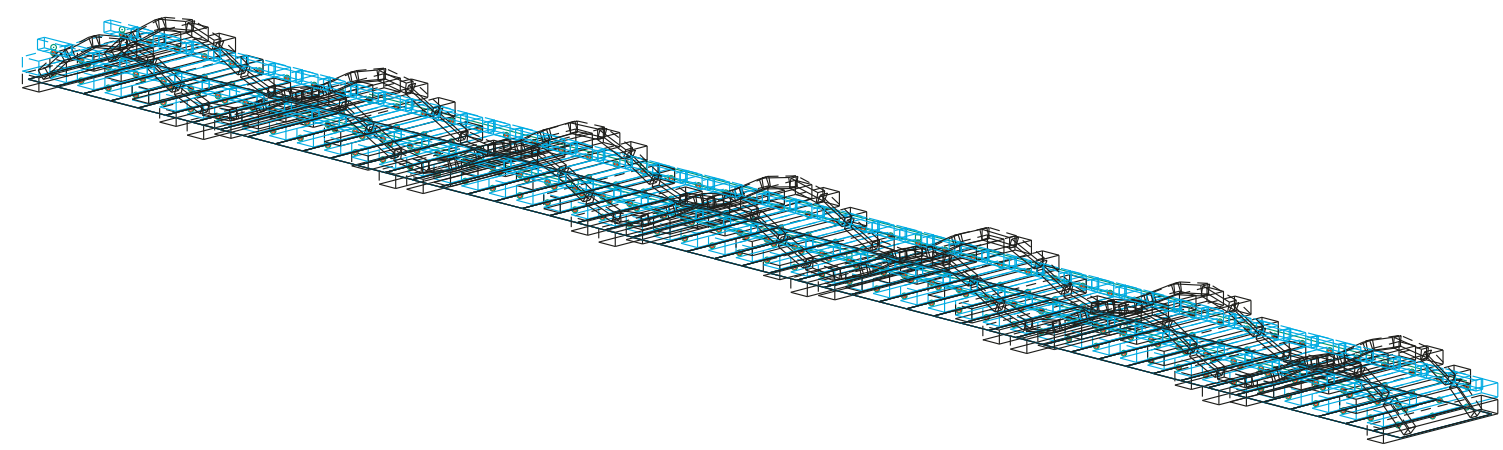

(a)

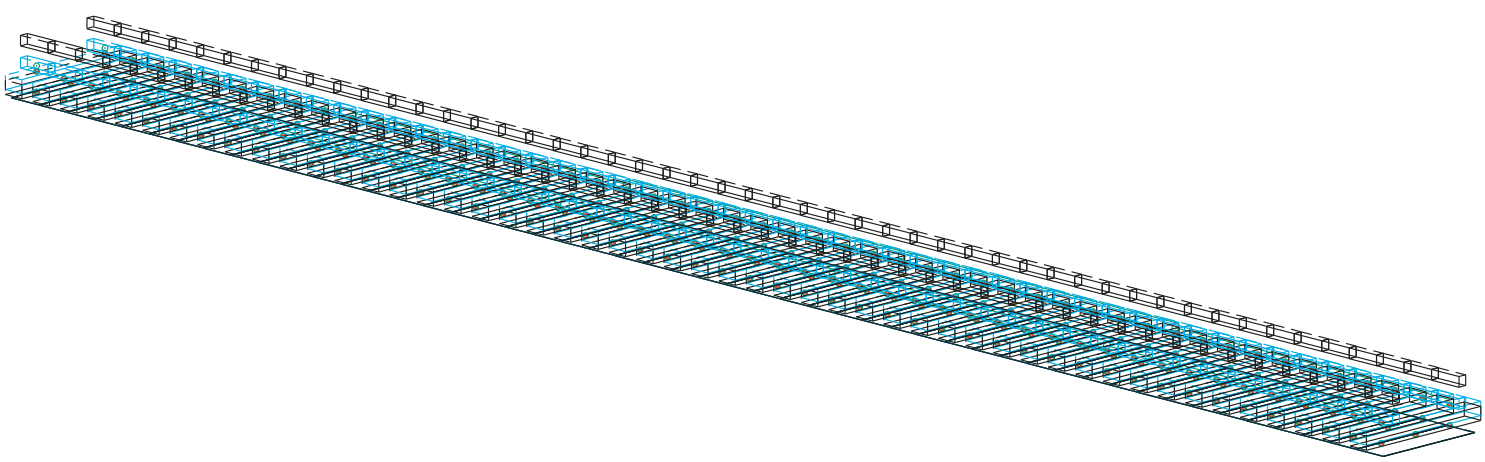

(b)

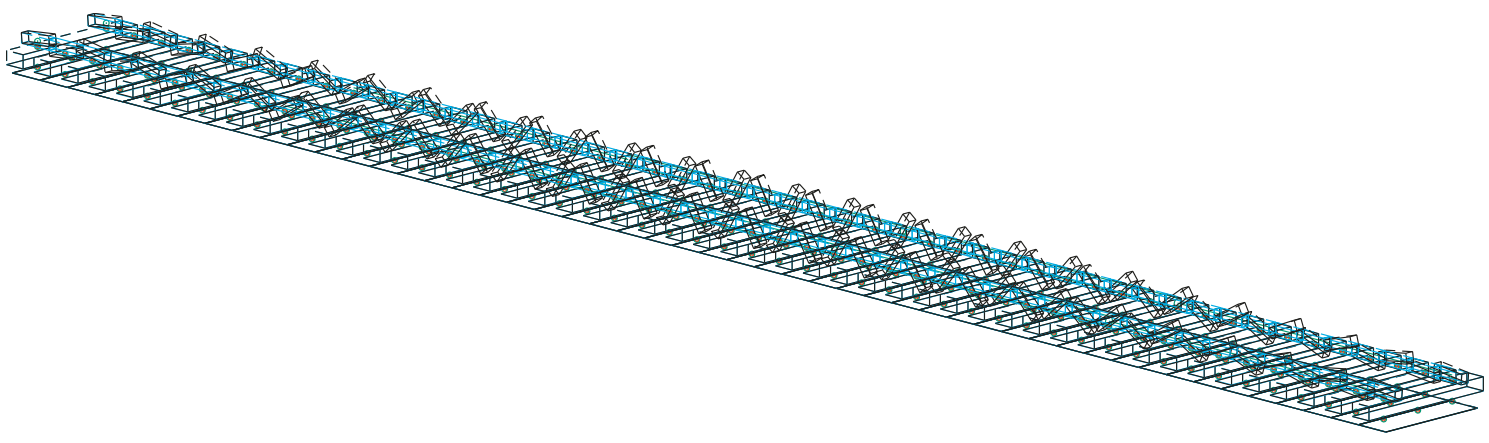

(c)

Figure 3: Mode shapes: (a) $129.10 \mathrm{~Hz}$, (b) $505.08 \mathrm{~Hz}$, and (c) $830.48 \mathrm{~Hz}$.

occurs in the frequency range of 200 to $600 \mathrm{~Hz}$, is captured at the frequency around $485 \mathrm{~Hz}$. In this model, pin-pin resonances can also be captured and is at the frequency around $900 \mathrm{~Hz}$.

Due to the approximation method (7)-(9), the magnitude of the receptance function on the rail between two sleepers is slightly lower compared to the receptance function on the rail above the sleeper. This is because the displacement and rotation of the rail masses adjacent to the point of observation do not always have the same magnitude, and the compromise of the magnitudes leads to slight reduction of the overall magnitude. In addition, the magnitude of the receptance at the pin-pin frequency when the receptance function is observed on the rail between the two sleepers is not as drastic as stated in the literature. This is because the approximation is based on the deflection and rotation of the two adjacent rail masses at the point of observation, and the projection is restricted by the rotation magnitude of the rail masses.
A modal analysis of the track as shown in Figure 3 is performed to illustrate the corresponding mode shapes of the resonance frequencies. The resonance frequencies from the modal analysis coincide well with the principal resonance frequencies computed from the track receptance tests despite the linearization of the track model in the modal analysis. Therefore, it is concluded that the track properties are valid to be applied to a more sophisticated turnout model.

In addition, a receptance test is performed on a moving mass track model as shown in Figure 4 to achieve a comparable track model with the full-track model. The moving mass track model consists of two rail masses coupled to a sleeper mass with a spring-damper element, and the sleeper mass is connected to rigid ground with two spring-damper elements. Besides, the sleeper mass is also attached to a rigid wall in the lateral direction using a spring-damper element with the value of $30 \mathrm{MN} / \mathrm{m}$ and $270 \mathrm{kNs} / \mathrm{m}$, respectively. Apart from the vertical translation, the rail masses and sleeper masses are constrained in every direction. 


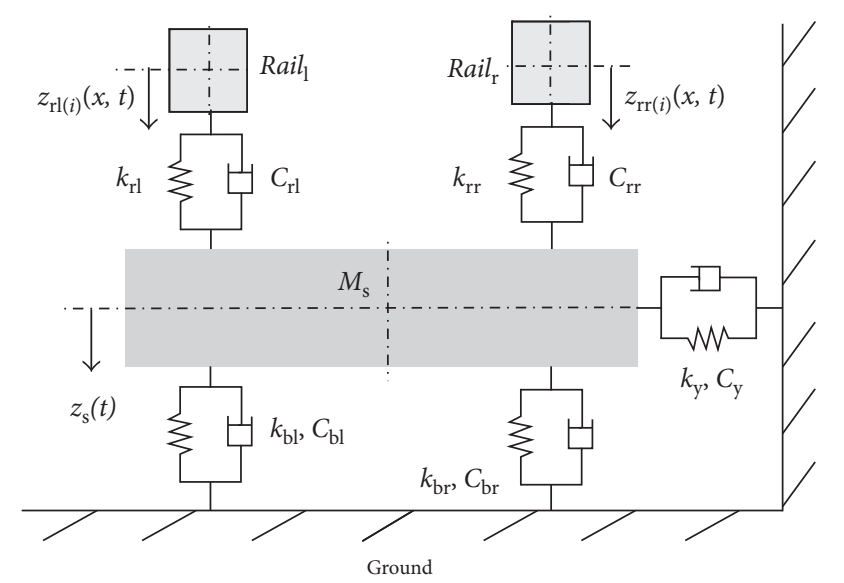

Figure 4: Moving mass track model.

It can be seen that the track receptance function of the moving mass track model when similar values in Table 1 are adopted appears nearly the same as the receptance functions from the full-track model. However, the pin-pin resonance in the moving mass track model is missing because of the absence of the beam element. Moreover, one should also note that when the moving mass model is connected to a train model, the second resonance frequency will not appear because the inertia of the rail masses will not be considered.

3.3. Turnout Model. The turnout model is a right turn 60E1$760-1: 15$ (nominal rail profile 60E1, curve radius $760 \mathrm{~m}$, and turnout angle $1: 15$ ) turnout without rail inclination. The variation of the rail profile in the turnout is accounted for by 30 rail cross sections along the turnout. The total length of the turnout model is $120 \mathrm{~m}$. The first $60 \mathrm{~m}$ of the track is a straight track modelled to damp the initial transient effect and the rest is the turnout model. This means that the turnout model consists of 400 rail masses, 200 sleeper masses, and 1000 spring-damper elements for the coupling of the rails to the sleepers and to the rigid ground.

Unlike normal track, the rail bending stiffness is varied according to the rail profile in contact with the wheels along the turnout. However, due to the constraints of the EulerBernoulli beam equation, the bending stiffness can only be varied sectionally. The bending stiffness of the beam sections is approximated by the mean of the bending stiffness at the closest adjacent measured rail profiles according to the construction drawing. In addition, the sleeper properties such as mass, dimension, and moment of inertias are also varied along the turnout according to the construction drawing.

Since the rails are positioned differently on the sleepers along the switch and crossing for facing and diverging route, the rails have to be positioned accordingly in the model for different moving directions (only diverging route is considered in this work). The rest of the track properties follow the validated track properties as listed in Table 1. Figure 5 is a schematic plan view of the turnout model without the $60 \mathrm{~m}$ straight section.

\section{Train-Turnout Coupling}

For the coupling of the train and turnout model, a stiff springdamper system $141 \mathrm{MN} / \mathrm{m}$ and $30 \mathrm{kNs} / \mathrm{m}$, respectively, is adopted for each wheel of the train connected to a neglected inertia fictitious mass. The fictitious masses are needed in order to extract wheel-rail profile contact information and any track irregularity during the time simulation, that is, offline lookup table is used in the model. The fictitious mass is connected to the Euler-Bernoulli beams by a very stiff "railrail" contact stiffness $k_{\mathrm{c}}$, which has a value of $5000 \mathrm{MN} / \mathrm{m}$ assuming the wheel and the rail do not lost contact during the simulation.

For the normal wheel-rail contact problem, Hertz contact theory is adopted. The creepages and creep forces are solved by using the FASTSIM algorithm by Kalker based on the simplified theory of rolling contact. Unlike moving mass track model, the inertia of the rail masses does not need to be neglected because the wheel of the train in the full-track model rolls over the track without carrying the track under the wheel.

\section{Numerical Results}

Two simulations have been carried out, train-turnout coupled model and moving mass turnout model. Similar train model and values up to wheel-rail contact are used for the both simulations. The train travelled through the turnout at the speed of $80 \mathrm{~km} / \mathrm{h}$. The switch panel of the turnout starts at $0 \mathrm{~m}$, and the crossing panel starts at around $47 \mathrm{~m}$. The sampling frequency for both simulations is $1000 \mathrm{~Hz}$.

Figure 6 shows the vertical normal force of the wheels on the leading wheelset. It can be seen that the vertical normal force is very similar in general for the both models. However, variation can be observed in switch and crossing panels where the dynamic impact is higher. This is probably because the track reacted to the dynamic impact differently and eventually affects the magnitude of the vertical normal force. Similar phenomena can be observed in the lateral normal force as illustrated in Figure 7. This can also be explained by the similarity of the vertical and lateral normal forces along the turnout when the dynamic interaction is not as significant. Figure 8 shows the zoomed-in vertical normal force. Sleeper passing effect can also be simulated in the trainturnout coupled model but not the moving mass turnout model.

Simulating the train-turnout coupled dynamic using this approach, additional information in connection to the track such as the time history of the sleeper, force exerted on the sleeper, sleeper acceleration, and force exerted on the ballast can also be observed. Figure 9 illustrates the forces from the left and the right rail exerted on the sleeper numbers 2, 40, and 80 near the switch panel, closure panel, and crossing panel, respectively. All the four wheel passages can be observed in the sleeper number 2 and number 40. However, only first two wheel passages can be observed at the sleeper number 80 due to the length of the track and the trailing bogie did not travel through it before the end of the simulation. It can be seen that the distribution of the forces is 


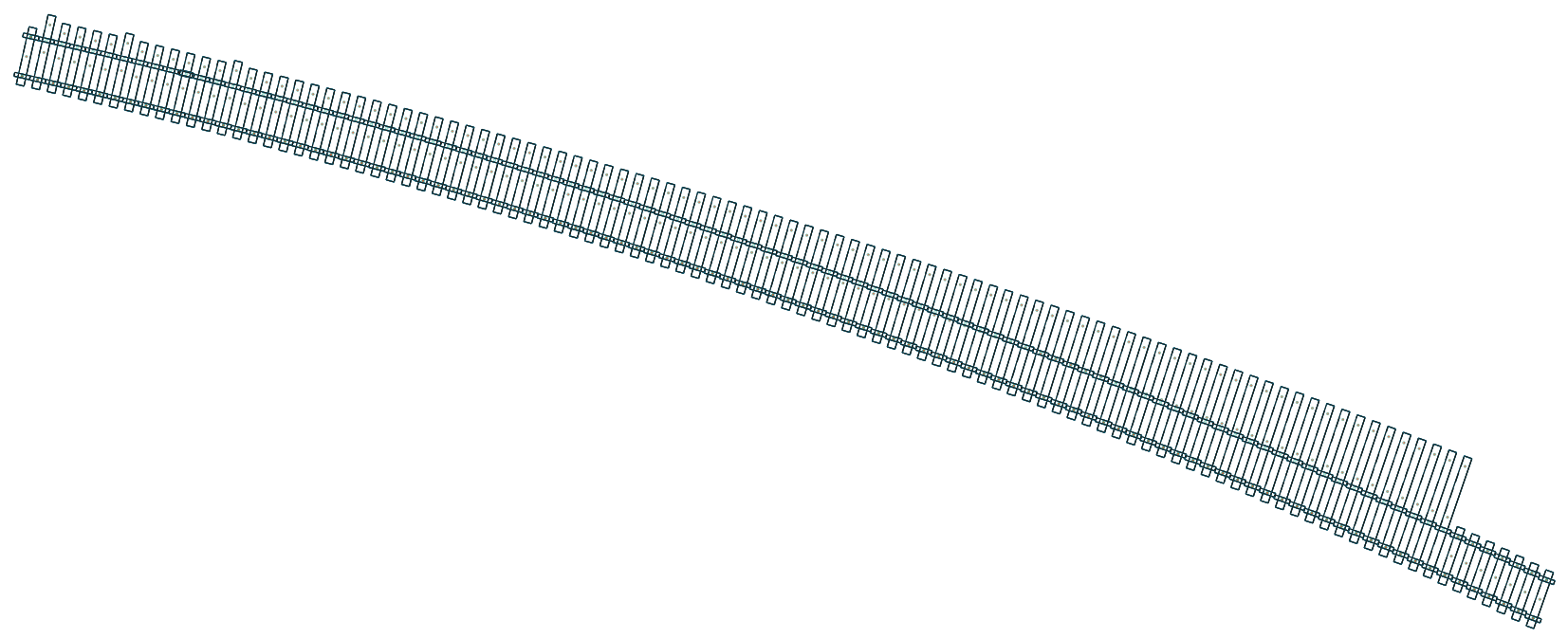

Figure 5: Schematic plan view of the turnout geometry.

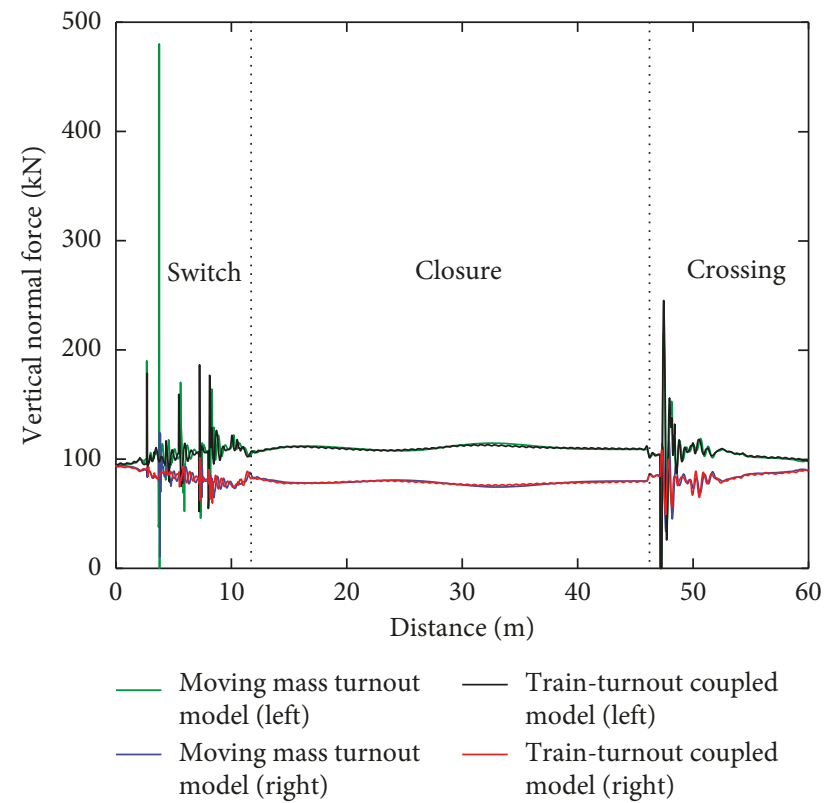

FIgURE 6: Vertical normal force of the wheels on the leading wheelset.

even for the first two wheel passage on the sleeper number 2, but the force gradually distributed more to the left wheel when the rear wheels passed the sleeper because of curvature experienced by the train. The curvature effect becomes more obvious at the sleeper number 40 . In addition to the curvature effect, the dynamic impact due to the crossing profile in the sleeper number 80 can be observed.

Figure 10 shows the maximum force exerted on the sleepers throughout the turnout. It can be seen that the maximum force exerted on the left rail is higher than that on the right rail because of the curvature of the track. Besides, highest force exerted on the sleeper is in the switch panel (sleeper number 1 to 20) and the lowest force exerted on the sleeper is in the crossing panel (sleeper number 70 to 90 ). This is because the bending stiffness of the rail is higher in crossing panel compared to the switch panel. The high

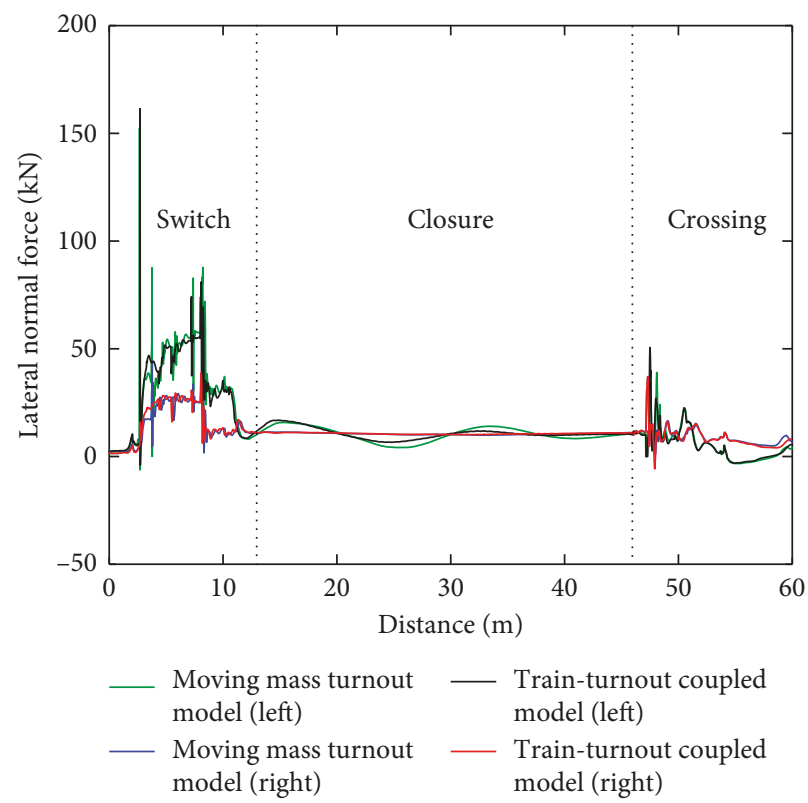

Figure 7: Lateral normal force of the wheels on the leading wheelset.

bending stiffness of the rail in crossing panel prevents the force from transferring down to the sleepers even though the dynamic impact is high in this region. Although the dynamic impact on the rail in the switch panel is not as significant as in the crossing panel, the maximum force exerted on the sleepers is higher compared to the force exerted on the sleeper in the crossing panel due to the relatively lower bending stiffness of the rail.

Figure 11 illustrates the forces transfer from the sleepers down to the ballast through the left, middle, and the right spring-damper elements under sleeper 2, 40, and 80 . It can be seen that the force under the sleepers on the right is higher than the middle and the left in general. This is because of the formation of the turnout in which the rails are placed gradually rightward on the sleepers along the diverging route. This effect is more obvious at the sleeper number 80 , which is 


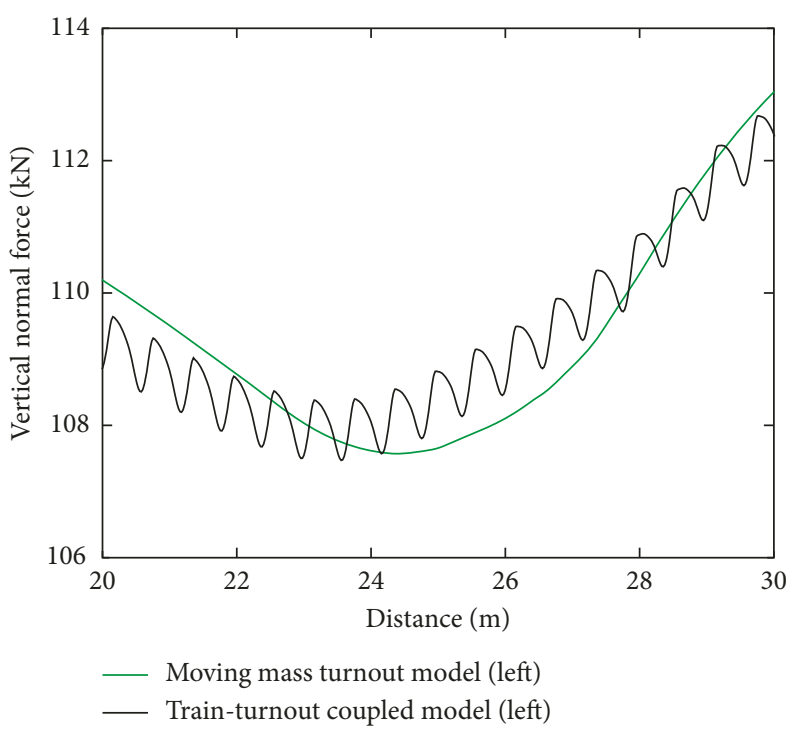

FIgURE 8: Sleeper passing frequency.

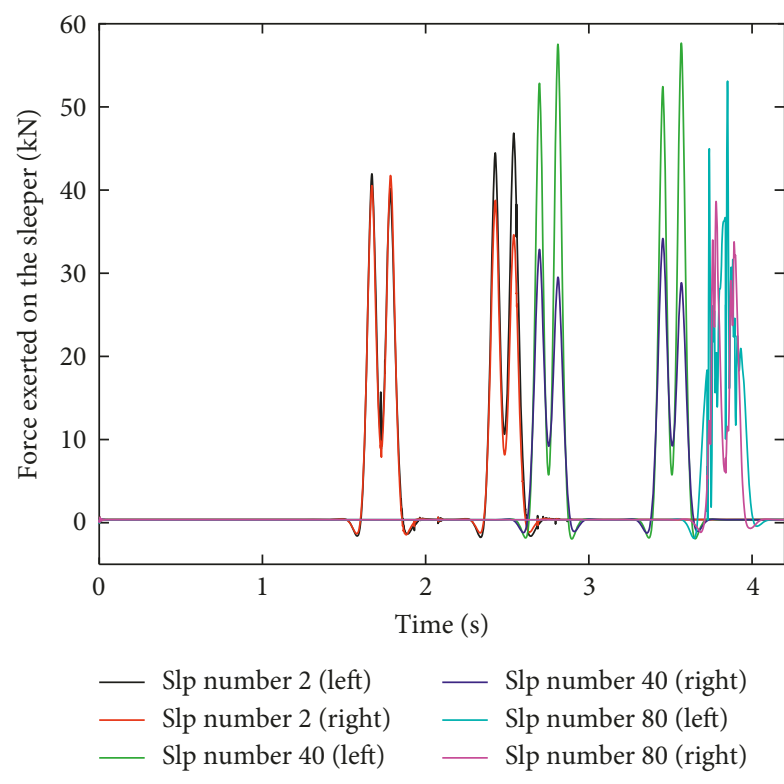

Figure 9: Force exerted on the sleeper.

near the crossing panel. The force exerted on the ballast on the right is greater than the force exerted on the ballast in the middle even though the magnitude of the impact force on the left rail (nearer to the middle of the sleeper) above the sleeper number 80 is higher. In addition, the magnitude of the initial force exerted on the ballast is also different along the turnout due to the variation of the sleeper masses along the turnout. The variation of the sleeper masses will also have effect towards the dynamic of the track in general. This is especially crucial if the condition under the track is of interest such as track settlement prediction. It is because the mass of the sleeper might affect the magnitude of the dynamic vertical force exerted on the ballast and eventually affect the accuracy of the track settlement prediction especially in the switch and crossing panels where the dynamic effect is significant.

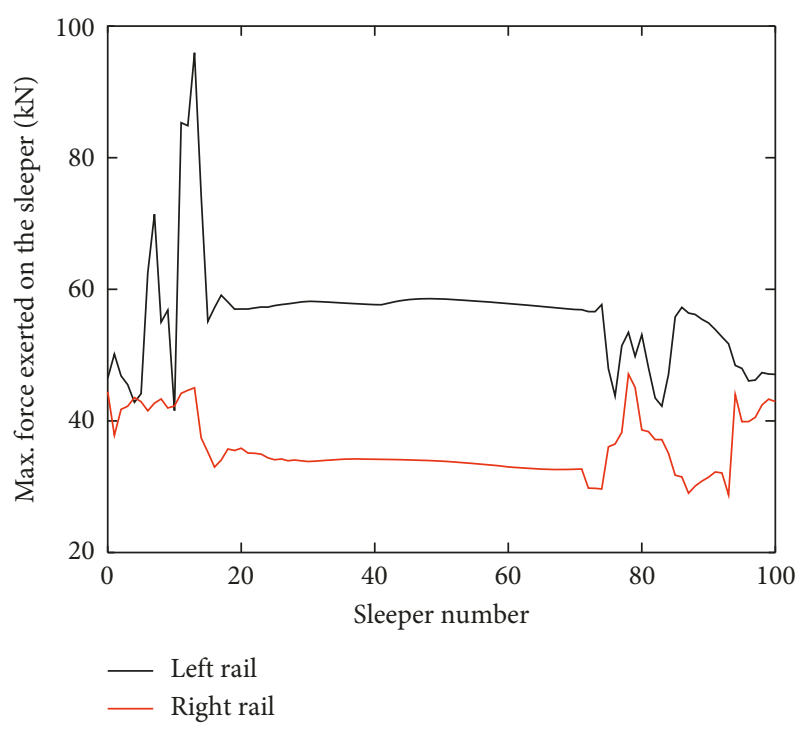

Figure 10: Maximum force exerted on the sleeper.

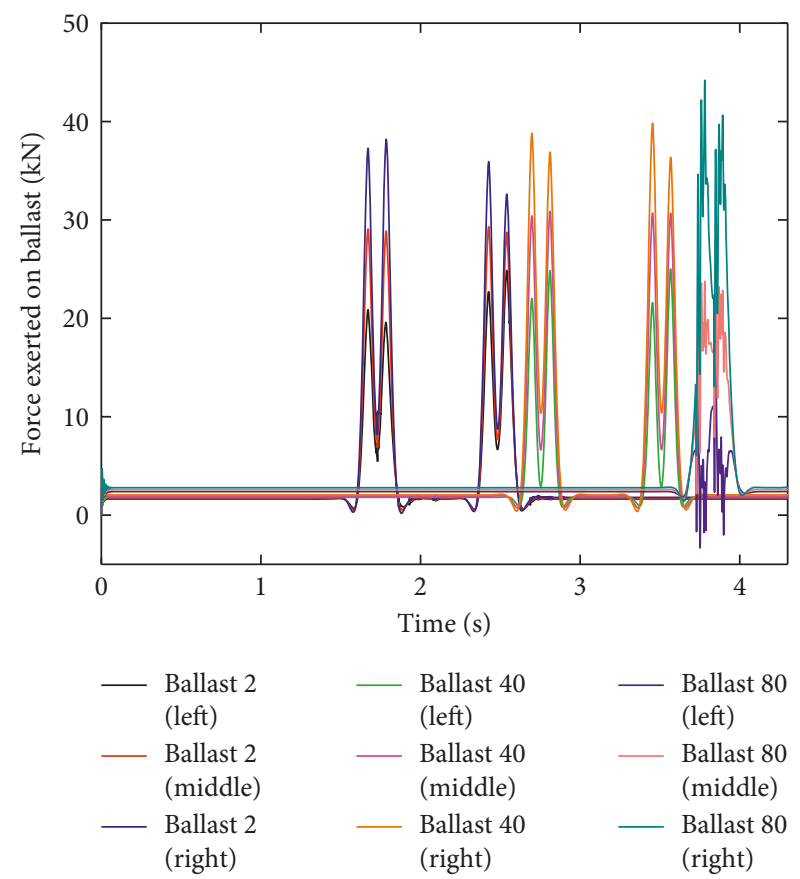

FIGURE 11: Force exerted on the ballast.

Figure 12 shows the maximum force exerted on the ballast along the turnout. The maximum force exerted on the ballast on the left, middle, and right started at around the same magnitude $30 \mathrm{kN}$. Due to the formation of the turnout as mentioned earlier, the maximum force exerted on the ballast on the right increases gradually and vice versa for the left. However, the maximum force exerted on the ballast in the middle does not fluctuate as much as the left and the right. Coincidently, at the sleeper number 13, the maximum force exerted on the ballast for all three points is very similar because of the balance of curvature and turnout formation effect. In addition, due to the change of the sleeper geometry back to the ordinary geometry started from sleeper number 93 , a reversal 


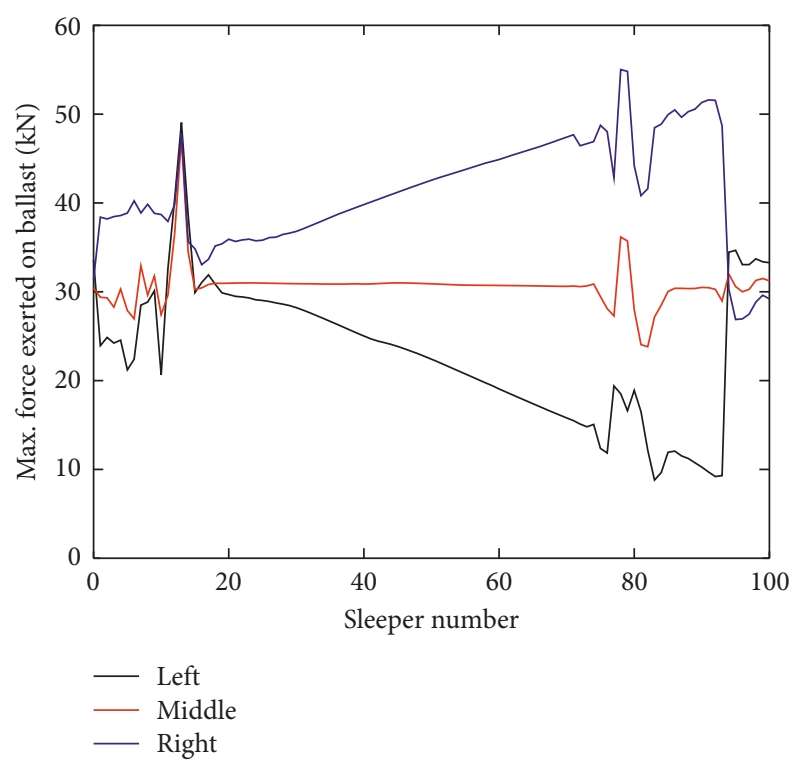

Figure 12: Maximum force exerted on the ballast.

effect of the left and the right maximum force exerted on the ballast due to the carbody roll can also be observed.

As a comparison, the force exerted on the sleeper and the ballast of the leading wheelset for moving mass turnout model is also plotted, Figure 13. It can be seen that the time history for the vertical normal force, the force exerted on the sleeper, and the force exerted on the ballast are very similar. This is because the vertical normal force is transmitted down to the sleeper and to the ground without affecting by the rail bending stiffness, discretisation of the track, and the formation of the turnout. The forces exerted on the ballast have a slightly higher magnitude compared to the forces exerted on the sleeper due to the existence of the sleeper mass. However, this variation is constant throughout the turnout due to the consistency of the sleeper mass in the moving mass model.

\section{Discussion}

In most of the cases, railway track is not the major focus in MBS train-track dynamic analysis. This is because MBS tools dedicated for train-track dynamic analysis are mainly used for the train dynamic analysis and the dynamics contributed by the track is relatively low, assuming that the track geometry is more or less consistent such as tangent and curve tracks. However, when a more sophisticated track structure such as switch and crossing is involved, the track effects such as rail bending stiffness and sleeper mass become crucial in order to simulate both train and track dynamics more accurately. The advantages of modelling railway track in MBS using this approach are listed as below:

(1) Train and track models can be modelled as a whole.

(2) Rail bending stiffness can be modelled and varied.

(3) Sleeper mass and track stiffness can be varied.

(4) Sleeper passing effect can be modelled.

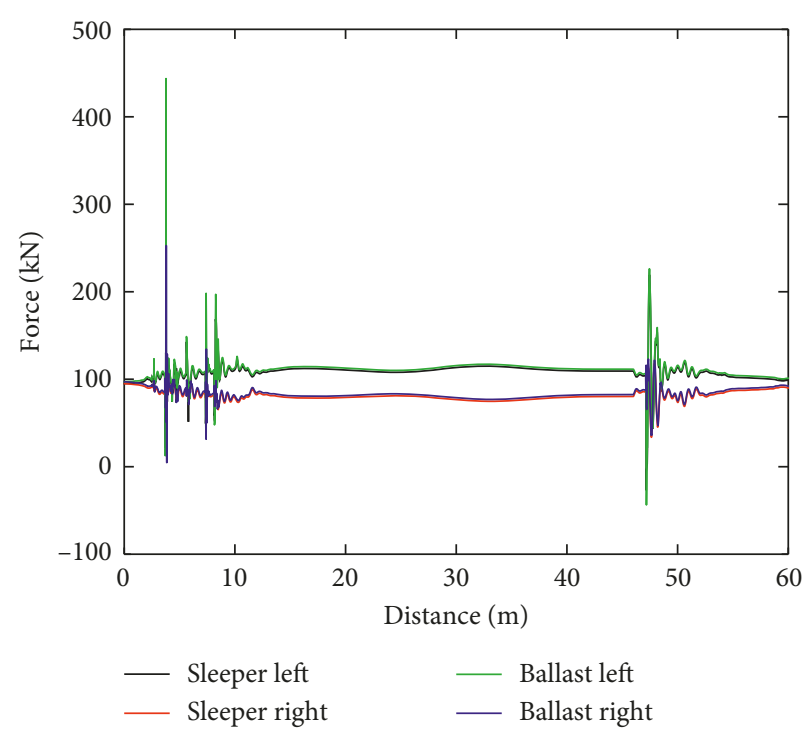

FIGURE 13: Force exerted on the sleeper and the ballast (moving mass turnout model).

(5) Dynamic interaction within the track can be computed and observed.

In addition, modelling railway track in MBS can make other type of analysis in the track, that is, settlement analysis, inclusion of ballast characteristic, hanging sleeper effect, track geometry irregularity, and track component optimisation, possible without oversimplifying the train or the track.

The downside of this approach is that generally in MBS, the initiation time increases with the increase of number of bodies in the simulation. For a switch and crossing model, many additional bodies are needed; for example, in this paper, additional 400 rail and 200 sleeper masses are needed alongside with the seven bodies for the train model. The initiation of the train-track model took 15 minutes, and the simulation took 11 minutes for a computer with processor Intel ${ }^{\circledR}$ Core $^{\mathrm{TM}}$ i7-4600 CPU @ $2.10 \mathrm{GHz}$ (4 CPUs) compared to the moving mass turnout model which took 3 minutes in total to simulate.

For that, a separate algorithm has been written to avoid the repetitive initiation process in which the initiation of the train-track model is saved during the first simulation, so that for the subsequent simulation using the same train-track model, the initiation of the model can be avoided.

\section{Conclusion}

In this paper, a train-turnout coupled dynamics has been simulated using a multibody simulation software GENSYS. Track parameters such as rail bending stiffness, rail bending stiffness variation, and sleeper mass variation along the turnout are considered in the model. The dynamic track properties of the turnout model such as rail masses' pitch moment of inertia, railpads' stiffness and damping, and ballast's stiffness and damping are validated beforehand by 
comparing the receptance functions from a straight track model to the receptance functions recorded in the literature.

After that, the results of the train-turnout coupled model are compared to the results of a moving mass train-turnout model. The following conclusions are drawn:

(1) The vertical and lateral normal forces for the trainturnout coupled model are in good agreement with moving mass train-turnout model.

(2) The full-turnout model has influence towards the vertical and lateral normal forces especially in the switch panel and crossing panel where the dynamic impact is higher.

(3) The force exerted on the sleepers of the train-turnout coupled model is lower compared to the moving mass track model because of the rail bending stiffness. Besides, the magnitude of the force exerted on the sleepers decreases with the increase of the rail bending stiffness.

(4) The force exerted on the ballast is position of the trainload dependent. For example, in the present paper, the force on the right side of the sleepers is greater along the turnout as the train is travelling through the diverging direction.

(5) The time history of the individual track component in the train-track coupled model can be analysed but not the moving mass track model.

It is also concluded that such approach can potentially provide a better train-track interaction result compared to moving mass track model because of the consideration of the track parameters. The future work is to validate the trainturnout model with a real turnout model by receptance test or comparing the components, that is, sleeper or rail accelerations from the real turnout with the numerical model.

\section{Conflicts of Interest}

The authors of this paper declared that there are no conflicts of interest in their submitted paper.

\section{Acknowledgments}

The present work was carried out at the Department of Civil and Environmental Engineering, Norwegian University of Science and Technology, and was funded by the Norwegian Railway Directorate. Mr. Ingemar Persson is also gratefully acknowledged for assisting and advising modelling in GENSYS.

\section{References}

[1] C. Alves, A. Paixão, E. Fortunato, and R. Calçada, "Under sleeper pads in transition zones at railway underpasses: numerical modelling and experimental validation," Structure and Infrastructure Engineering, vol. 11, no. 11, pp. 1432-1449, 2015.

[2] M. Wiest, E. Kassa, W. Daves, J. C. O. Nielsen, and H. Ossberger, "Assessment of methods for calculating contact pressure in wheel-rail/switch contact," Wear, vol. 265, no. 910, pp. 1439-1445, 2008.
[3] A. Johansson, B. Pålsson, M. Ekh et al., "Simulation of wheel-rail contact and damage in switches \& crossings," Wear, vol. 271, no. 1-2, pp. 472-481, 2011.

[4] A. Paixão, E. Fortunato, and R. Calçada, "A numerical study on the influence of backfill settlements in the train/track interaction at transition zones to railway bridges," Proceedings of the Institution of Mechanical Engineers, Part F: Journal of Rail and Rapid Transit, vol. 230, no. 3, pp. 866-878, 2015.

[5] H. Wang, V. Markine, and I. Shevtsov, "The analysis of degradation mechanism in track transition zones using $3 \mathrm{~d}$ finite element model," in Proceedings of the Second International Conference on Railway Technology: Research, Development and Maintenance, Civil-Comp Press, Stirlingshire, UK, 2014.

[6] E. Kassa and J. C. O. Nielsen, "Dynamic interaction between train and railway turnout: full-scale field test and validation of simulation models," Vehicle System Dynamics, vol. 46, no. 1, pp. 521-534, 2008.

[7] R. Lagos, A. Alonso, J. Vinolas, and X. Pérez, "Rail vehicle passing through a turnout: analysis of different turnout designs and wheel profiles," Proceedings of the Institution of Mechanical Engineers Part F: Journal of Rail and Rapid Transit, vol. 226, no. 6, pp. 587-602, 2012.

[8] C. Wan, V. L. Markine, and I. Y. Shevtsov, "Improvement of vehicle-turnout interaction by optimising the shape of crossing nose," Vehicle System Dynamics, vol. 52, no. 11, pp. 1517-1540, 2014.

[9] B. Pålsson, "Design optimisation of switch rails in railway turnouts," Vehicle System Dynamics, vol. 51, no. 10, pp. 1619-1639, 2013.

[10] A. Lau and E. Kassa, "Simulation of vehicle-track interaction in small radius curves and switches and crossings," in Proceedings of the Third International Conference on Railway Technology: Research, Development and Maintenance, CivilComp Press, Stirlingshire, UK, 2014.

[11] C. Esveld, Modern Railway Track, MRT-Productions, Zaltbommel, Netherlands, 2001.

[12] J. Evans and M. Berg, "Challenges in simulation of rail vehicle dynamics," Vehicle System Dynamics, vol. 47, no. 8, pp. 1023-1048, 2009.

[13] W. Zhai and Z. Cai, "Dynamic interaction between a lumped mass vehicle and a discretely supported continuous rail track," Computers and Structures, vol. 63, no. 5, pp. 987-997, 1997.

[14] K. L. Knothe and S. L. Grassie, "Modelling of railway track and vehicle/track interaction at high frequencies," Vehicle System Dynamics, vol. 22, no. 3-4, pp. 209-262, 1993.

[15] G. Diana, F. Cheli, S. Bruni, and A. Collina, "Dynamic interaction between rail vehicles and track for high speed train," Vehicle System Dynamics, vol. 24, no. S1, pp. 15-30, 1995.

[16] N. Ottosen and H. Petersson, Introduction to the Finite Element Method, Prentice-Hall, Upper Saddle River, NJ, USA, 1992.

[17] Y. Sun, C. Cole, M. Spiryagin, and M. Dhanasekar, "Vertical dynamic interaction of trains and rail steel bridges," Electronic Journal of Structural Engineering, vol. 13, no. 1, pp. 88-97, 2013.

[18] S. Iwnicki, "Manchester benchmarks for rail vehicle simulation," Vehicle System Dynamics, vol. 30, no. 3-4, pp. 295-313, 1998.

[19] S. Iwnicki, Handbook of Railway Vehicle Dynamics, CRC Press, Boca Raton, FL, USA, 2006. 


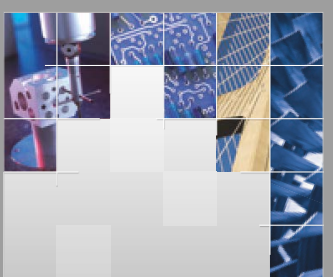

\section{Enfincering}
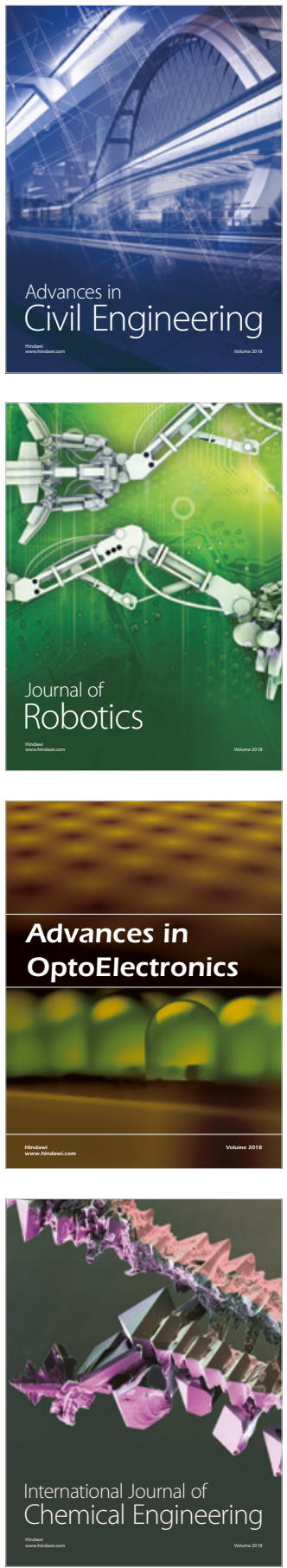

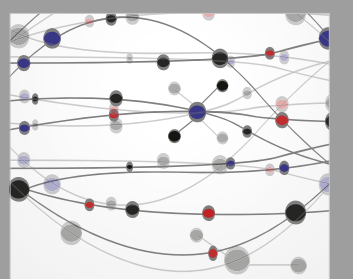

\section{Rotating \\ Machinery}

The Scientific World Journal

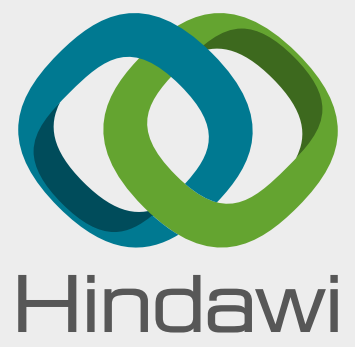

Submit your manuscripts at

www.hindawi.com
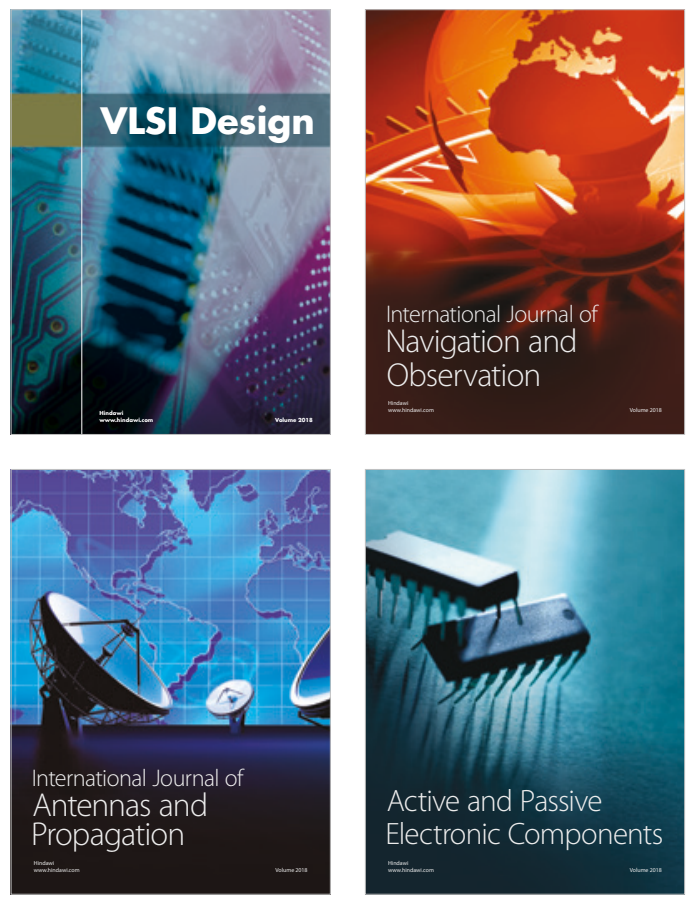
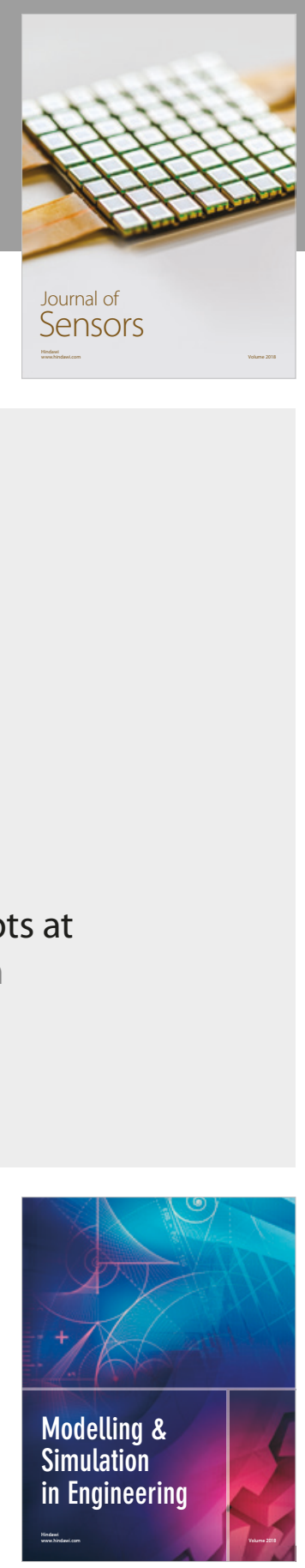

\section{Advances \\ Multimedia}
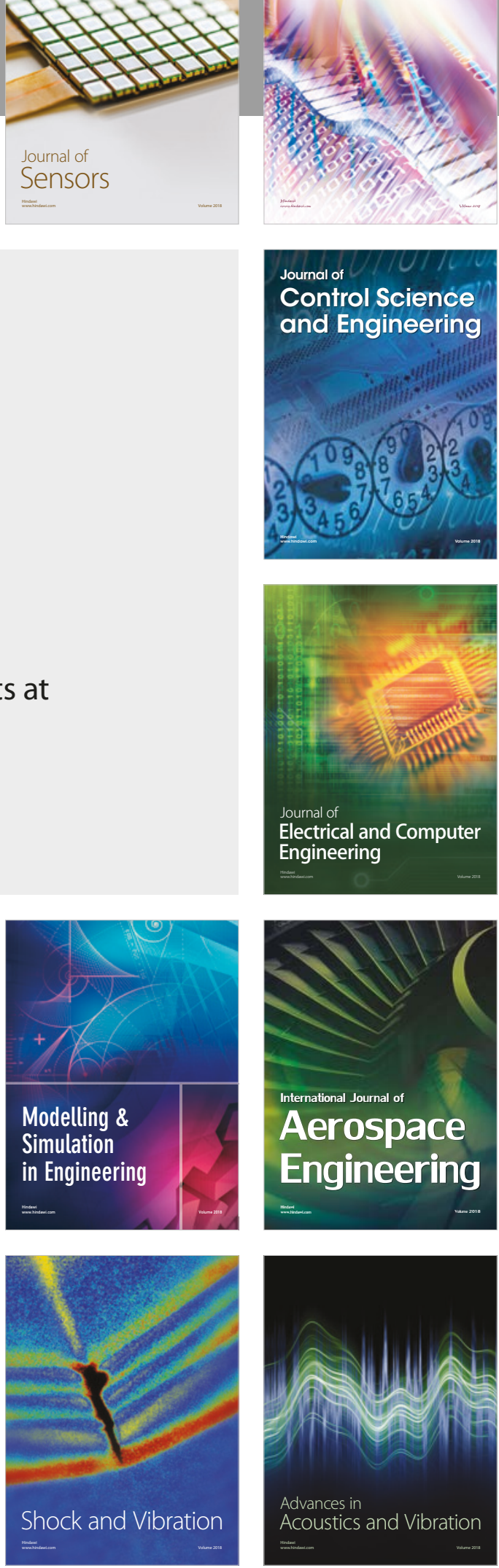